\title{
Die europäische Verfassungskrise und die Strategie des ,langen Atems'
}

\author{
Daniel Göler und Mathias Jopp*
}

Das Scheitern der Referenden in Frankreich und den Niederlanden über den Europäischen Verfassungsvertrag jährt sich demnächst - und immer noch zeichnet sich keine Lösung der derzeitigen Krise ab. Die von den Staats- und Regierungschefs im Juni 2005 ausgerufene Denkpause brachte zunächst wenig Konkretes und mutete - um ein im Europaparlament kursierendes Bonmot aufzugreifen - teilweise eher als ,Pause vom Denken' an. Seit der Vereinbarung über den europäischen Finanzrahmen im Dezember 2005 kommt jedoch wieder Bewegung in die Diskussion. Dies wird nicht zuletzt daran deutlich, dass europäische Spitzenpolitiker vermehrt ihre unterschiedlichen Vorstellungen über mögliche Auswege aus der Krise skizzieren. ${ }^{1}$ Systematisiert man die verschiedenen Lösungsvorschläge, kristallisieren sich im Wesentlichen vier , Obergruppen' von Einzelvorschlägen heraus, die sich danach unterscheiden, ob sie den Verfassungsvertrag, den Vertrag von Nizza, beide gemeinsam oder aber Formen der flexiblen Integration als Ausgangspunkt ihrer Überlegungen nehmen. ${ }^{2}$

Kompliziert wird die Lage dadurch, dass die konkreten Vorschläge häufig eine Mischung aus verschiedenen Modellen darstellen und sich in Stellungnahmen derselben Personen divergierende Schwerpunktsetzungen wiederfinden. Deshalb wäre es wenig zweckmäßig, die Lösungsvorschläge nach bestimmten Vertretern zu ordnen. Stattdessen wird im Folgenden das Tableau an Optionen entsprechend den substantiellen Reformansätzen betrachtet, auch wenn diese Einzelvorschläge - abgesehen von den Initiativen, die sich für eine vollständige oder modifizierte Inkraftsetzung des Verfassungsvertrages aussprechen - in der aktuellen Debatte meist nicht isoliert vorgebracht, sondern mit den anderen Vorschlägen zu Maßnahmenbündeln kombiniert werden. Diese inhaltliche Systematisierung bietet den Vorteil, dass die Grundgedanken der einzelnen Lösungsansätze klarer hervortreten und sich auch deren Realisierungschancen und die mit ihnen zusammenhängenden Probleme besser aufzeigen lassen. Deutlich wird hierbei zum einen, dass jede Strategie zur Lösung der europäischen Verfassungskrise mit Risiken behaftet ist, zum anderen aber die Anzahl der ,Gleichungen mit mehreren Unbekannten' im Hinblick auf das erzielbare Ergebnis im Falle eines völlig neuen Reformanlaufs aller Wahrscheinlichkeit nach zunimmt.

* Dr. Daniel Göler, wissenschaftlicher Mitarbeiter am Institut für Europäische Politik, Berlin. Lehrbeauftragter an der Europa-Universität Viadrina (Frankfurt/Oder).

Dr. Mathias Jopp, Direktor des Instituts für Europäische Politik, Berlin.

Die Autoren danken Frau Kathrin Brockmann, MA, für ihre Mithilfe bei der Recherche.

1 Vgl. Angela Merkel: Regierungserklärung vor dem Deutschen Bundestag vom 30. November 2005, Bundestags-Plenarprotokoll, 16. Wahlperiode, 4. Sitzung, S. 88-89; Jacques Chirac: Déclaration (Europäischer Rat 15./16. Dezember 2005), abrufbar unter: http://www.elysee.fr; Tony Blair: Speech on future of Europe, Oxford, 2. Februar 2006, abrufbar unter: http://www.pm.gov.uk/output/Page9003.asp; Nicolas Sarkozy: Voeux à la presse, 12. Januar 2006, abrufbar unter: http://www.u-m-p.org/; Guy Verhofstadt: Die Vereinigten Staaten von Europa. Manifest für ein neues Europa, Eupen 2006; Polen will mit der Reform der EU ganz vorne beginnen, in: Frankfurter Allgemeine Zeitung vom 8. März 2006.

2 Eine ähnliche, wenn im Detail auch andere Systematisierung findet sich bei Udo Diedrichs/Wolfgang Wessels: Die Europäische Union in der Verfassungsfalle. Analysen, Entwicklungen und Optionen, in: integration 4/ 2005, S. 293-306. 


\section{Festhalten am Verfassungsvertrag}

Obwohl die Verfassung schon vom niederländischen Außenminister Bot als tot bezeichnet wurde, ${ }^{3}$ ist ihre Inkraftsetzung nach wie vor eine Option, die vor allem vom Europäischen Parlament ${ }^{4}$ und den Ländern propagiert wird, die den Verfassungsvertrag bereits ratifiziert haben, wobei sich die Bundesregierung besonders hervorgetan hat. ${ }^{5}$ Dem liegt die Überzeugung zu Grunde, dass der Verfassungsvertrag das Optimum des derzeit Machbaren ist. Die meisten anderen Denkmodelle würden gewissermaßen zurück zu ,square one' führen und wieder von vorne die mühsamen und aufwändigen zwischenstaatlichen Abstimmungsprozesse bis zum Ende einer Regierungskonferenz erfordern, um das, europäische Reformrad' neu zu erfinden. Auf der anderen Seite kann der Verfassungsvertrag aber nur in Kraft treten, wenn er von allen 25 Mitgliedstaaten ratifiziert wird, deren Staats- und Regierungschefs ihn ja bereits unterschrieben haben. Allerdings haben die gleichen Staats- und Regierungschefs in Vorahnung möglicher Ratifizierungsprobleme vereinbart, ,dass der Europäische Rat befasst wird, wenn nach Ablauf von zwei Jahren nach der Unterzeichnung des Vertrages über eine Verfassung für Europa vier Fünftel der Mitgliedstaaten den genannten Vertrag ratifiziert haben und in einem oder mehreren Mitgliedstaaten Schwierigkeiten bei der Ratifikation aufgetreten sind" . ${ }^{6}$ Dies lässt einen gewissen Spielraum für ,kreative Lösungen' erkennen, seien es nun erneute Ratifizierungsanläufe oder begrenzte Opt-out-Regelungen für einzelne Länder. ${ }^{7}$ Voraussetzung wäre allerdings die Ratifizierung des Verfassungsvertrages durch mindestens 20 Mitgliedstaaten.

\section{Stand und Entwicklungspotenzial des Ratifizierungsprozesses}

Bis heute haben insgesamt 14 Mitgliedstaaten den Ratifizierungsprozess abgeschlossen. ${ }^{8}$ Zudem wurde das Ratifizierungsgesetz in Estland Anfang Februar 2006 zur zweiten Lesung in das Parlament eingebracht, wo eine Zustimmung als sicher gilt. ${ }^{9}$ Damit hätten in naher Zukunft 15 Staaten den Verfassungsvertrag angenommen und zwei abgelehnt. Ob das Erreichen der 20er Schwelle realistisch ist, hängt somit zunächst von den acht Staaten ab, die den Ratifizierungsprozess momentan ausgesetzt haben. Dabei lassen sich drei Gruppen unterscheiden:

- Länder, in denen die Ratifizierung parlamentarisch erfolgt (Finnland und Schweden)

- Länder, die sich auf ein Referendum verständigt haben oder in denen ein Referendum vorgeschrieben ist (Dänemark, Großbritannien, Irland und Portugal)

- Länder, in denen ein Referendum sehr wahrscheinlich, die endgültige Entscheidung hierüber aber noch nicht getroffen ist (Tschechien und Polen).

3 Vgl. David Rennie: EU Constitution is Dead, Says Dutch Minister, in: Daily Telegraph vom 12. Januar 2006.

4 Vgl. European Parliament: The Period of Reflection. The Structure, Subjects and Context for an Assessment of the Debate on the European Union (2005/2146(INI)), abrufbar unter: www.europarl.eu.int. Damit liegt das Europäische Parlament auf einer Linie mit seiner auch in der Vergangenheit immer wieder zu Tage getretenen verfassungsbefürwortenden Sicht. Vgl. hierzu unter anderem auch die verschiedenen Plädoyers prominenter Europaparlamentarier zum Verfassungsvertrag in: Mathias Jopp/Saskia Matl (Hrsg.): Der Vertrag über eine Verfassung für Europa. Analysen zur Konstitutionalisierung der EU, Baden-Baden 2005, S. 535-591.

5 Vgl. Merkel, Regierungserklärung, S. 88.

6 Vgl. Erklärung zur Ratifikation des Vertrages über eine Verfassung für Europa (Nr. 30).

7 Vgl. Jörg Monar: Optionen für den Ernstfall. Auswege aus einer möglichen Ratifizierungskrise des Verfassungsvertrages, in: integration 1/2005, S. 20.

8 Vorbehaltlich möglicher Entscheidungen einzelner Verfassungsgerichte.

9 Vgl. Estonia Begins Ratifying EU Constitution, in: EU Business vom 8. Februar 2006. 
Eine Annahme der Verfassung ist in der ersten Gruppe am wahrscheinlichsten, zumal sich sowohl in Finnland als auch in Schweden eine breite parlamentarische Mehrheit für den Verfassungsvertrag abzeichnet. Für den weiteren Fortgang des Verfassungsprozesses stellt sich damit vor allem die Frage, ob sich beide Länder zu einer baldigen Entscheidung bewegen können, von der dann wiederum eine positive Dynamik auf die übrigen ,Nachzügler' ausgehen könnte. Bei den vier Ländern, in denen ein Referendum vorgesehen ist, stellt sich die Lage hingegen komplexer dar. Während es in Großbritannien eine relativ konstante Mehrheit gegen den Verfassungsvertrag gibt, die nach den Referenden in Frankreich und den Niederlanden noch weiter zugenommen hat, ${ }^{10}$ sahen die Meinungsumfragen in Dänemark zunächst günstiger aus. Allerdings wich dort die anfängliche Zustimmung nach den negativen Referenden einer knappen Ablehnung, wenngleich ein relativ hoher Anteil von 28 Prozent der Befragten sich weder auf ein Ja noch ein Nein festlegte; ${ }^{11}$ auch sollte man die demoskopischen Pendelausschläge nach dem französisch-niederländischen, Doppel-Schock' nicht überbewerten. In Irland sieht die Situation demgegenüber günstiger aus. Zwar wich auch dort die anfängliche Zustimmung nach den negativen Voten in Frankreich und den Niederlanden einer knappen Ablehnung, allerdings verbesserte sich die Stimmung im Herbst 2005 so, dass nach einer Umfrage die Befürworter wieder deutlich vorne lagen, wenn auch der Anteil von 50 Prozent unentschiedener Wähler außerordentlich hoch ist und damit keine sicheren Schlussfolgerungen über den Ausgang eines Referendums gezogen werden können. ${ }^{12} \mathrm{Ob}$ die irische und dänische Bevölkerung die Verfassung letztlich bestätigt oder ablehnt, dürfte von der dann aktuellen europapolitischen Stimmungslage und den innenpolitischen Rahmenbedingungen abhängen. Deutlich stabiler ist die Situation in Portugal. Hier spricht sich seit langem eine Mehrheit der Bevölkerung für den Verfassungsvertrag aus. Zwar schmolz diese Mehrheit Mitte letzten Jahres auf 50,8 Prozent, ${ }^{13}$ allerdings standen diese Umfrageergebnisse unter dem unmittelbaren Eindruck der gescheiterten Referenden in Frankreich und den Niederlanden. Sollte die auf unbestimmte Zeit verschobene Abstimmung nicht noch von unerwarteten innenpolitischen Auseinandersetzungen überlagert werden, ist ein positiver Ausgang des Referendums in Portugal durchaus zu erwarten.

Am unübersichtlichsten ist die Situation hingegen in den Ländern, welche sich noch nicht endgültig auf die Form der Ratifizierung festgelegt haben: In Polen trifft man auf die besondere Situation, dass ein Referendum, das andernorts eine zusätzliche Hürde darstellt, die Wahrscheinlichkeit der Ratifizierung eher erhöht. Denn auch wenn die Zahl der Verfassungsbefürworter in den letzten beiden Jahren gesunken ist, übersteigt diese immer noch die der Gegner. So ergab eine Umfrage vom Juni 2005 (nach dem Scheitern der Referenden) eine Zustimmung von 43 Prozent, 24 Prozent lehnten die Verfassung ab und 33 Prozent waren unentschieden. ${ }^{14}$ Eine parlamentarische Ratifizierung würde hingegen aller Voraussicht nach scheitern, da die europaskeptischen Parteien bei den Sejm-Wahlen Ende letzten Jahres die Mehrheit errungen haben. ${ }^{15}$ In Tschechien sah die Situation bis Mai 2005 ähnlich aus: Während es in der Bevölkerung eine Mehrheit für den Verfassungsvertrag gab, war eine par-

10 Vgl. Katrin Pecker: Der Stand der Ratifizierungsprozesse zur EU-Verfassung in den Mitgliedstaaten. IEP Ratifizierungssurvey, Berlin 2005, S. 9, abrufbar unter: http://www.iep-berlin.de/publik/iep-policy-brief/index.htm.

11 Für den Verfassungsvertrag votierten $34 \%$, dagegen $38 \%$. Vgl. Institut für Europäische Politik (Hrsg.): EU 25-Watch, Nr. 2, abrufbar unter: http://www.iep-berlin.de/publik/EU25-Watch/index.htm, S. 43.

12 Die genauen Werte waren: $37 \%$ für und $13 \%$ gegen den Verfassungsvertrag; $50 \%$ waren unentschieden. Vgl. Ireland Publishes Discussion Paper on EU Constitution, in: EU Business vom 13. Oktober 2005.

13 Vgl. Wallström rechnet nicht mit Referendum in Dänemark, Spiegel Online vom 10. Juni 2005.

14 Vgl. Institut für Europäische Politik, EU 25-Watch, S. 84.

15 Vgl. ebenda. 
lamentarische Ratifizierung eher unwahrscheinlich, da die vorgeschriebene 60-ProzentHürde in beiden Parlamentskammern angesichts der Ablehnung von ODS und Kommunisten nicht erreichbar schien. Nach den Referenden in Frankreich und den Niederlanden ist die öffentliche Unterstützung in Tschechien allerdings eingebrochen. Im Juli 2005 sprachen sich nur noch 21 Prozent für und 37 Prozent gegen die Verfassung aus, ${ }^{16}$ der Rest war unentschlossen. Damit sind dort die Chancen einer erfolgreichen Ratifizierung gesunken.

Zusammenfassend lässt sich sagen, dass in den acht Ländern, in denen die Ratifizierung zur Zeit ausgesetzt ist, die Chancen für eine Annahme in Portugal, Schweden und Finnland als gut einzuschätzen sind. Um die in der Erklärung zur Ratifizierung des Verfassungsvertrages vereinbarte Zahl von 20 Mitgliedstaaten zu erreichen, würden demnach noch zwei Staaten benötigt. Da ein positives Votum der Bevölkerung in Großbritannien auf absehbare Zeit nicht $\mathrm{zu}$ erwarten und auch in Tschechien eine Ratifizierung nach heutigem Stand eher unwahrscheinlich ist, wird es letztlich darauf ankommen, wie der Ratifikationsprozess in Irland, Dänemark und Polen verläuft. Da in den beiden ersten Ländern, in denen ein Referendum zwingend vorgeschrieben ist, der Anteil der unentschlossenen Wähler sehr hoch ist, lassen sich zur Zeit keine eindeutigen Prognosen stellen. Angesichts der deutlichen Mehrheit der Befürworter in Irland und der nur knappen Mehrheit der Verfassungsgegner in Dänemark wäre allerdings in einer innenpolitisch günstigen Situation in beiden Ländern ein positiver Ausgang denkbar. In Polen ist die Situation noch komplexer, da vor dem Hintergrund der aktuellen Umfragewerte und der noch nicht endgültig entschiedenen Form der Ratifizierung positive Voten ebenso denkbar sind wie eine Ablehnung. Hierbei könnte sich der Sieg der europakritischen Parteien bei den Sejm-Wahlen paradoxerweise sogar als Chance erweisen. Denn die Geschichte europapolitischer Referenden zeigt, dass ablehnende Voten zu einem großen Teil aus der Unzufriedenheit mit der jeweiligen nationalen Regierung resultieren, die durch ein negatives Stimmverhalten abgestraft werden soll. ${ }^{17}$ Wenn aber nun die Regierung selbst der Verfassung kritisch gegenübersteht, könnte der Mechanismus einer informellen Koalition aus Regierungs- und Europagegnern unwirksam werden. Wie dem auch sei, das Erreichen der 20er Schwelle wird zwar nicht einfach, aber auch keineswegs unmöglich sein.

\section{Vollständige Annahme des Verfassungsvertrages mittels ergänzender Erklärungen oder Protokolle}

Allerdings wäre auch mit dem Erreichen der 20er Schwelle das Problem der Ratifizierung keineswegs gelöst. Denn nach wie vor müsste ein gangbarer Weg für die Staaten gefunden werden, welche die Verfassung nicht ratifiziert haben oder vor Schwierigkeiten stehen werden. Die in der Wissenschaft angedachte Lösung, dass die betreffenden Staaten aus der EU austreten beziehungsweise über den Umweg einer Neugründung auf der Basis des Verfassungsvertrages faktisch ausgeschlossen werden, scheidet als realistisches Szenario aus, ${ }^{18}$ zumal mit Frankreich und den Niederlanden zwei für den Integrationsprozess unverzichtbare Staaten betroffen wären. Demnach müsste man Mittel und Wege finden, mit denen vor allem Frankreich, aber auch die Niederlande und eventuell noch weitere Länder, in de-

16 Vgl. Gericht setzt Ratifizierung der EU-Verfassung aus, in: Frankfurter Allgemeine Zeitung vom 16. Juli 2005.

17 Vgl. Gesa-S. Kuhle: Schlussfolgerungen aus den gescheiterten Referenden zum Europäischen Verfassungsvertrag, IEP-Policy-Brief 2/2005, S. 1, abrufbar unter: http://www.iep-berlin.de/publik/iep-policy-brief/index.htm.

18 Eine detaillierte Auseinandersetzung mit den verschiedenen hierzu diskutierten Denkmodellen liefert Andreas Maurer: Austritt, Ausschluss oder institutionelle Anpassung. Optionen nach dem Scheitern des EU-Verfassungsvertrages, in: Internationale Politik und Gesellschaft 1/2005, S. 165-183; ders.: Die Ratifikation des Verfassungsvertrages, in: Jopp/Matl, Der Vertrag über eine Verfassung für Europa, S. 506. 
nen die Ratifizierung gefährdet ist, die Verfassung letztlich doch annehmen könnten. Hierbei ist zu berücksichtigen, dass es von führenden Politikern in Frankreich und den Niederlanden abgelehnt wird, der jeweiligen Bevölkerung denselben Vertrag in unveränderter Form nochmals zur Abstimmung vorzulegen. ${ }^{19}$

Bei der Suche nach Lösungswegen gibt es in der europäischen Integrationsgeschichte allerdings zwei Präzedenzfälle, in denen nach einem gescheiterten Referendum ein Vertrag mit einem zweiten Anlauf doch noch angenommen wurde: Dänemark beim Vertrag von Maastricht und Irland beim Vertrag von Nizza. Im ersten Fall gestand man Dänemark auf dem Europäischen Rat von Edinburgh im Dezember 1992 vor dem zweiten Referendum verschiedene Opt-out-Regelungen zu, die den beim ersten Referendum geäußerten Bedenken entgegenkamen und in einem von allen Mitgliedstaaten zu ratifizierenden Protokoll festgehalten wurden. ${ }^{20} \mathrm{Im}$ zweiten Fall legte man der irischen Bevölkerung zwar tatsächlich denselben Vertrag nochmals zur Abstimmung vor, die allerdings durch eine doppelte Maßnahme flankiert war: Zum einen wurden innenpolitisch die Beteiligungsrechte des irischen Parlaments in der Europapolitik erhöht und zum anderen gab die irische Regierung auf dem Europäischen Rat von Sevilla eine Erklärung zur Europäischen Sicherheits- und Verteidigungspolitik und irischen Neutralität ab. Diese wurde durch eine entsprechende Erklärung des Europäischen Rates ergänzt, welche die nationale Erklärung Irlands zur Kenntnis nahm und deren Inhalte bestätigte. ${ }^{21}$ Faktisch stellten beide Erklärungen zwar nichts anderes dar als eine Bestätigung des Status quo; sie waren allerdings in der zweiten Referendumskampagne insoweit wichtig, als sie zuvor geäußerte Befürchtungen aufgriffen und klarstellten, dass die irische Neutralität durch die EU unberührt bleibt.

Für die gegenwärtige Lage kann das dänische Opt-out-Modell kaum Vorbild sein. Denn in den Niederlanden lassen sich keine klaren Kritikpunkte am Verfassungsvertrag erkennen, die durch Opt-out-Regelungen ausgeräumt werden könnten. In Frankreich wird zwar immer wieder die Kritik an der als zu , unsozial' empfundenen europäischen Wirtschaftsordnung als zentraler Grund für die Ablehnung genannt, allerdings stellt sich hier das Problem, dass diese Frage kaum durch Opt-outs gelöst werden kann: Denn zum einen zielen die Erwartungen in weiten Teilen der französischen Bevölkerung eher auf mehr als auf weniger Europa (vor allem als Schutzraum vor Globalisierung), zum anderen würden denkbare wirtschaftspolitische Opt-outs den Kernbereich des Binnenmarktes und damit ein Grundelement des gesamten Integrationsprozesses schwächen. Anders sieht es hingegen bei der Möglichkeit ,klarstellender Erklärungen' und der Variante inhaltlicher Ergänzungsprotokolle aus.

Die aktuelle Diskussion konzentriert sich daher vor allem auf die Möglichkeit einer sozialpolitischen Erklärung, ein Vorschlag, der von Bundeskanzlerin Merkel im Dezember letzten Jahres wohlwollend aufgenommen wurde. ${ }^{22}$ Bei allen Initiativen im sozialpolitischen Bereich stellt sich jedoch das Grundproblem, dass eine substantielle Ausweitung der sozialen Dimension zwar die Widerstände in Frankreich verringern, diese dafür aber in anderen Staaten wie Großbritannien vergrößern dürfte. Damit bliebe nur die Möglichkeit eines eher deklaratorischen Dokuments, das im Prinzip lediglich alle im Verfassungsvertrag ohnehin schon enthaltenen sozial- und beschäftigungspolitisch relevanten Regelungen zusammen-

19 So jüngst nochmals Nicolas Sarkozy am 16. Februar 2006 bei einem Vortrag der Konrad-Adenauer-Stiftung und der Deutschen Gesellschaft für Auswärtige Politik in Berlin, abrufbar unter: www.botschaft-frankreich.de, S. 5.

20 Vgl. Protokoll Nr. 5 des EU-Vertrages (Maastricht) über die Position Dänemarks.

21 Vgl. Europäischer Rat (Sevilla): Schlussfolgerungen des Vorsitzes, 21./22. Juni 2002, Anlage III und IV.

22 Vgl. hierzu den Bericht von Nikolaus Blome über die deutsche Europapolitik: Steinbrück droht mit Kürzung der EU-Regionalhilfen, in: Die Welt vom 29. Dezember 2005. 
fasst und gegebenenfalls durch einige gezielte Initiativen wie etwa den in der letzten finanziellen Vorausschau schon beschlossenen Globalisierungsfonds ergänzt. Ob eine solche Erklärung ausreichen würde, den Vorbehalten der Bürger entgegenzukommen, hängt im Wesentlichen davon ab, wie diese von den politischen Eliten, verkauft' wird, und bleibt damit abzuwarten.

Der Sache nach ähnlich, in der Form allerdings zu unterscheiden, ist die Variante eines Sozialprotokolls. Ein solches Protokoll hätte den Vorteil, dass es als integraler Bestandteil dem Vertrag hinzugefügt würde, womit das Bedürfnis der Bürger nach einem sozialen Europa deutlich sichtbarer aufgegriffen werden könnte. Allerdings stellen sich auch in diesem Fall alle zuvor genannten Probleme in verschärfter Form. Denn wenn es darum geht, Positionen zur sozialen Dimension als Protokoll, das Primärrechtsstatus hat, neu zu formulieren, könnten etwaige Widerstände noch stärker werden als im Falle einer entsprechenden Erklärung. Von daher ließe sich auch bei einem solchen Sozialprotokoll lediglich der Status quo zusammenfassen, jedoch mit dem entscheidenden Nachteil, dass bei der Protokollform etwaige Kontroversen heftiger ausgetragen würden. Allerdings könnte der Akzeptanzgrad eines solchen Protokolls bei den Mitgliedstaaten unter Umständen erhöht werden, wenn es von vornherein mit dem Gedanken der flexiblen Integration (siehe unten) verbunden und als Opt-in-Protokoll ${ }^{23}$ - mit moderaten, den Status quo leicht ergänzenden Elementen - dem Vertrag hinzugefügt würde. Zwar müsste auch ein solches Opt-in-Protokoll nochmals von allen Mitgliedstaaten ratifiziert werden, was im Vergleich zu dem ,Erklärungs-Modell' eine zusätzliche Verkomplizierung beinhaltete; es beträfe aber nur die im Protokoll genannten oder sich diesem in einer gesetzten Frist anschließenden Länder, was die Chancen einer Ratifizierung durch alle Mitgliedstaaten verbesserte.

In welcher Form auch immer man die Wünsche nach einer Betonung der sozialen Dimension aufgreift würde dies primär den französischen Befindlichkeiten entgegenkommen. Denn in den Niederlanden spielt der Bereich , soziales Europa' eine weitaus geringere Rolle. Allerdings gab es dort mit der Nettobeitragszahler-Diskussion und der Angst vor einem nationalen Souveränitätsverlust während des Referendums zwei Ablehnungsgründe, an denen sich ansetzen ließe. Der Erste ist durch die neue Finanzielle Vorausschau, die auf der Ebene des Europäischen Rates im Dezember letzten Jahres nach mühevollen Verhandlungen verabschiedet wurde, bereits entschärft worden. Zusätzlich könnte man im Rahmen der Revisionsklausel einen generellen Korrekturmechanismus einführen. Und auch für den Bereich der Souveränitäts- und Identitätsverlust-Ängste ließen sich gezielte Initiativen denken. In diesem Zusammenhang ist es - auch wenn es manchmal belächelt wird - gar nicht so abwegig von der österreichischen Präsidentschaft, eine Diskussion über die kulturelle Identität der Europäischen Union zu führen wie jüngst mit der Tagung „The sound of Europe“ in Salzburg. ${ }^{24}$ Da die Frage der Nettobeitragslasten ohnehin außerhalb des Primärrechts liegt hier wurden die Kritikpunkte in der niederländischen Abstimmung in der Tat unberechtigter Weise auf den Verfassungsvertrag projiziert - und Fragen der kulturellen Identität eher über konkrete Projekte denn Vertragsartikel zu regeln wären, würden sich auch hier gezielte Reformen außerhalb des Primärrechts beziehungsweise entsprechende Erklärungen anbieten.

Das Erklärungs-Modell oder die Protokoll-Varianten hätten unter Umständen den Vorteil, auf erneute Referenden verzichten zu können und eine parlamentarische Ratifizierung

23 Die Idee eines Opt-in-Protokolls ist nicht neu. Sie wurde während der Verhandlungen über den Vertrag von Amsterdam erstmals im ,6-Nations-Proposal“ ausformuliert - allerdings für die Verteidigungspolitik und die in diesem Bereich willigen EU-Staaten, eine gegenseitige Beistandsverpflichtung einzugehen.

24 Vgl. http://www.austria.gv.at. 
anstreben zu können. In Frankreich wie den Niederlanden wäre dies juristisch absolut möglich, da Referenden nicht zwingend vorgeschrieben sind (und in den Niederlanden ohnehin nur einen konsultativen Charakter haben) ${ }^{25}$ Die betreffenden Regierungen müssten dann in die Richtung argumentieren, dass man die Kritik der Referenden verstanden und durch Nachverhandlungen mit den Partnerregierungen Konsequenzen gezogen habe, die in schriftlich fixierter (möglicherweise völkerrechtlich verbindlicher) Form dem Vertrag hinzugefügt werden. Allerdings wäre ein solcher Weg aufgrund der auf ein Referendum verpflichteten gegenwärtigen Regierungen nur nach einem Regierungswechsel gangbar, wobei die Wahlen in Frankreich und den Niederlanden im nächsten Jahr diesbezüglich eine Chance sein könnten. Während in den Niederlanden nur sehr verhalten und vereinzelt über Möglichkeiten einer parlamentarischen Ratifizierung nachgedacht wird, lassen die öffentlichen Äußerungen des UMP-Vorsitzenden Sarkozy aufhorchen, dass in Frankreich das Referendum nun, da die Amtszeit des Präsidenten auf fünf Jahre verkürzt wurde, nicht mehr die gleiche Bedeutung wie früher habe. ${ }^{26}$ Problematisch bei dem Weg ergänzender Erklärungen oder Protokolle ist jedoch, dass diese auch auf mögliche Ablehnungsgründe in denjenigen Staaten abgestimmt sein müssten, in denen die Ratifizierung noch aussteht. Nicht zuletzt aufgrund der dort teilweise noch ungeklärten Form der Ratifizierung (parlamentarisch oder per Referendum) liegen hier entsprechende Ansatzpunkte zu einem gewissen Teil noch im Bereich des Spekulativen.

Allerdings wäre die Perspektive einer Lösung der französischen Ratifizierungsproblematik für sich schon ein großer Gewinn für die mehrheitliche Gruppe der Verfassungsbefürworter unter den Mitgliedstaaten und würde ihrer Sache ohne Zweifel Auftrieb geben. Am Ende käme es darauf an, die Zahl der ,Verfassungsskeptiker' auf ein Minimum zu begrenzen, um erst dann zwischen diesen und den Verfassungsbefürwortern nach Lösungsmöglichkeiten zu suchen. Diese Strategie ist mühsam und zäh. Sie könnte auch nur dann funktionieren - bei einem nach wie vor offenen , end game' - wenn sich die These bewahrheitet, dass sich in den Ländern, in denen die Verfassung abgelehnt wurde, die Widerstände in der Hauptsache nicht gegen die Substanz des Verfassungsvertrages richteten.

\section{Erarbeitung und Aushandlung eines neuen Verfassungsvertrages}

Ausgangspunkt für die Forderung nach einer grundlegenden Überarbeitung des Verfassungsvertrages ist die Annahme, dass dieser in seiner jetzigen Form nicht mehr in Kraft treten kann. Angestoßen wurde die Debatte vor allem von den Europaparlamentariern Duff und Voggenhuber, die dafür plädierten, im Rahmen der Reflexionsphase die Verfassung grundsätzlich zu überarbeiten und hierzu einen neuen Konvent einzuberufen. ${ }^{27}$ Das Grundproblem hierbei ist, dass der Verfassungsvertrag nach einem 26-monatigen Deliberations- und Verhandlungsmarathon in Konvent und Regierungskonferenz in vielen Bereichen durchaus als Maximum des Erreichbaren angesehen werden kann. Auch gibt es keine ausreichenden Anhaltspunkte, die darauf schließen ließen, wie ein reformierter Verfassungsvertrag denn aussehen müsste, um auf eine größere Akzeptanz zu stoßen. Die Auswertung der demoskopischen Daten zeigt, dass es keine konkreten inhaltlichen Projekte der Verfassung gab, gegen die sich die Ablehnung richtete. ${ }^{28}$ Eher könnte man sagen, dass ein Großteil der Bürger in Frankreich noch weiter gehende Regelungen im Bereich der sozialen Dimension wünschten.

25 Vgl. Pecker, Stand der Ratifizierungsprozesse, S. 7 und 13-14.

26 Sarkozy stellt sein Programm vor, in: Frankfurter Allgemeine Zeitung vom 13. Januar 2006.

27 Vgl. Andrew Duff/Johannes Voggenhuber: Entwurf eines Berichts über die Reflexionsphase (2005/2146 (INI) - vorläufig - ), S. 7. 
Allerdings ist es unwahrscheinlich, dass dies in einem erneuten Verhandlungsmarathon erreichbar wäre. Auch gilt es zu bedenken, dass der Verfassungsvertrag eine Mischung aus optimal bewerteten Sachergebnissen und klassischen Kompromisslösungen darstellt. ${ }^{29}$ Eine Neuverhandlung ließe weder im ersten noch im zweiten Bereich grundsätzliche Veränderungen erwarten. Auch gibt es keine erkennbaren sachlichen Gründe, vor zweieinhalb Jahren inhaltlich für gut befundene Ergebnisse nun völlig anders zu bewerten. Wenn aber nicht erkennbar ist, dass eine Neuverhandlung ein grundsätzlich anderes Ergebnis produzieren würde, wäre die Erarbeitung eines neuen Verfassungsvertrages wenig hilfreich. Vielmehr bestünde die Gefahr, dass ein solcher Prozess im Rahmen eines neuen Konvents und/oder einer Regierungskonferenz sich über Jahre hinzieht, ohne bessere Ergebnisse zu erzielen, was die Akzeptanz eines solchen Vertrages durch die Bürger in einem neuen Ratifizierungsprozess eher verringerte als erhöhte.

\section{Nizza plus ...}

Die zweite Gruppe von Denkansätzen sieht nicht mehr die Verfassung, sondern den Vertrag von Nizza als Grundlage künftiger Reformschritte. Dabei wird neben entsprechenden Stellungnahmen von politischer Seite ${ }^{30}$ auch in der wissenschaftlichen Diskussion die Auffassung vertreten, dass man gut beraten wäre, ,den Verfassungsvertrag feierlich [zu] begraben" ${ }^{31}$ um sich kreativen Lösungsoptionen jenseits der Verfassung zuzuwenden. In der konkreten Ausgestaltung lassen sich im Wesentlichen drei Ansätze erkennen, mit denen die bestehenden Verträge weiterentwickelt werden sollen: Zum Ersten eine reine Reform der Institutionen, zum Zweiten eine umfassende Reform des gesamten Primärrechts (was nicht weit vom oben genannten Neuverhandeln des Verfassungsvertrags entfernt wäre) und zum Dritten eine einstweilige Beibehaltung des primärrechtlichen Status quo.

\section{Nizza plus Institutionenreform}

Den Vorschlag, sich nach dem Scheitern der Referenden in Frankreich und den Niederlanden dem Projekt einer institutionellen Reform zuzuwenden, kam als Erstes vom französischen Präsidenten Chirac, ${ }^{32}$ der hierzu als Ausgangspunkt nicht mehr den Verfassungsvertrag, sondern den Vertrag von Nizza heranzog: „Les Chefs d'État et de gouvernement puissent prendre les décisions nécessaires pour améliorer le fonctionnement des institutions en partant du cadre des traités existants. "33 Für den genauen Weg, auf dem eine solche institutionelle Reform erfolgen soll, gibt es grundsätzlich mehrere Optionen, die von den konkreten Einzelvorhaben abhängen: Die Neuordnung der Arbeitsweise des Rates ließe sich im Rahmen seines Selbstorganisationsrechts verwirklichen und auch die Reform der Kommission könnte ohne Vertragsrevision angegangen werden, da bereits Nizza den Übergang zu einem System der gleichberechtigten Rotation mit dem Beitritt des 27. Mitgliedstaates vorsieht. ${ }^{34}$ Nicht ohne Regierungskonferenz geändert werden könnten die Stimmgewichtung im

28 Vgl. Europäische Kommission: La Constitution européenne. Sondage post-référendum en France, Flash Eurobarométre 171/2005; Europäische Kommission: The European Constitution. Post-Referendum Survey in The Netherlands, Flash Eurobarometer 172/2005.

29 Grundsätzlich hierzu vgl. Daniel Göler: Deliberation - ein Zukunftsmodell europäischer Entscheidungsfindung. Analyse der Beratungen des Verfassungskonvents 2002-2003, Baden-Baden 2006, S. 318 ff.

30 Vgl. Rennie, EU Constitution is Dead.

31 Diedrichs/Wessels, Die Europäische Union in der Verfassungsfalle, S. 305.

32 Vgl. Chirac, Déclaration.

33 Jacques Chirac: Allocution lors de la présentation des vœux du Corps diplomatique, Paris, 10. Januar 2006, abrufbar unter: http://www.elysee.fr.

34 Vgl. Protokoll über die Erweiterung der Europäischen Union (Protokoll zum Vertrag von Nizza), Artikel 4. 
Ministerrat und die Zusammensetzung des Europäischen Parlaments. Allerdings bieten sich mit Blick auf beide Reformvorhaben gewisse Anpassungsmöglichkeiten anlässlich des in den nächsten Jahren anstehenden Beitritts von Kroatien. Denn sowohl die Sitzverteilung im Europäischen Parlament als auch die Stimmgewichtung im Ministerrat ist in den bisherigen Verträgen auf eine 27er Union ausgelegt. Da es insbesondere bei der Stimmgewichtung im Ministerrat nicht nur um das Stimmverhältnis zwischen den einzelnen Staaten, sondern auch den verschiedenen Staatengruppen (große und kleine Staaten) geht, könnte der Beitritt Kroatiens darüber hinaus der geeignete Anlass sein, das gesamte System der Stimmgewichtung und die Zusammensetzung des Europäischen Parlaments im Rahmen einer Regierungskonferenz zu überarbeiten.

Der Nachteil dieser Variante besteht darin, dass auch mit einer ,kleinen“ Regierungskonferenz die Pandorabüchse der schwierigen institutionellen Fragen wieder geöffnet würde und zudem eine reine Reform der Institutionen in eine ähnliche verhandlungstechnische Sackgasse münden könnte, wie dies bei der Regierungskonferenz von Nizza der Fall war. Gerade dort wurde deutlich, wie problematisch Verhandlungen über eine institutionelle Neuordnung sein können, wenn diese isoliert und nicht im Rahmen größerer Verhandlungspakete behandelt werden (auch beim Verfassungsvertrag ging es neben der Institutionenreform um ein Gesamtpaket, das die Grundrechte, Veränderungen in einzelnen Politikbereichen, die Rechtspersönlichkeit der EU, die Kompetenzordnung et cetera umfasste). Darüber hinaus würde die Trennung der institutionellen Reformen von den übrigen Verhandlungsmaterien auch die Kompromissbereitschaft einiger Staaten bei Letzteren senken. Vor diesem Hintergrund können die jüngsten Initiativen von Jacques Chirac, die von Nicolas Sarkozy aufgegriffen wurden, ${ }^{35}$ durchaus kritisch gesehen werden. Zwar fordern auch sie neben der Institutionenreform Verbesserungen in konkreten Politikbereichen, sei es innerhalb der bestehenden Verträge, durch gezielte Vertragsänderungen oder im Rahmen von Modellen der differenzierten Integration. ${ }^{36}$ Problematisch ist allerdings, dass bei ihnen beide Elemente institutionelle und inhaltliche Reform - als separate Vorhaben erscheinen, deren zeitliche Abfolge unklar ist. Sollte dies zu einer isolierten Behandlung der Institutionenfrage führen, würde dieser Vorschlag aber die genannten Probleme nach sich ziehen und die Gefahr einer Wiederholung von Nizza in sich bergen. Auch könnte die Strategie separater und zeitlich entzerrter Verhandlungen über verschiedene institutionelle oder politikbereichsspezifische Fragen zur permanenten Institutionalisierung der Regierungskonferenz führen, was weder der Stabilität des Integrationsprozesses dienen noch die Akzeptanz der Europäischen Union bei den Bürgern fördern dürfte.

\section{Umfassende Reform des Vertrags von Nizza}

Bei der Frage, wie man den Vertrag von Nizza grundlegend ändern müsste, fällt auf, dass viele Themen genannt werden, die bereits durch den Verfassungsvertrag aufgegriffen werden, wie die stärkere Einbeziehung der nationalen Parlamente, ${ }^{37}$ der Grundrechtsschutz sowie die auf den ersten Blick eher technisch anmutende, ihrer Bedeutung nach aber keineswegs zu unterschätzende Frage nach der Vereinfachung der Rechtsetzungsverfahren oder die Einführung einer klaren Normenhierarchie. Und auch in den Bereichen der konkreten Politikfelder weichen die Schwerpunkte nicht grundsätzlich von denen des Verfassungsvertrages ab: die

35 Vgl. Sarkozy, Vortrag vom 16. Februar 2006 in Berlin.

36 Vgl. ebenda; Chirac, Allocution lors de la présentation des vœux du Corps diplomatique.

37 Domenique de Villepin: Deutschland, Frankreich und die Zukunft der Europäischen Union, Berlin, 18. Januar 2006, abrufbar unter: www.botschaft-frankreich.de, S. 7. 
Effektivierung der Gemeinsamen Außen- und Sicherheitspolitik ${ }^{38}$ sowie der Ausbau der Zusammenarbeit im Bereich der Innen- und Justizpolitik (mit dem Schwerpunkt Terrorismusabwehr). Auch die nach der russisch-ukrainischen Gaskrise Anfang dieses Jahres vor allem von polnischer, ${ }^{39}$ aber auch von französischer und deutscher Seite ${ }^{40}$ vorgeschlagene intensivere Zusammenarbeit im Bereich der Energiepolitik findet eine Entsprechung in der Verfassung, die erstmals einen - wenn auch noch bescheidenen - Abschnitt zur Energiepolitik enthält. ${ }^{41}$

Besonders kritisch ist bei allen Vorschlägen zu einer weitreichenden Reform von Nizza das Verhältnis von institutionellen zu inhaltlichen Reformen. Zwar lässt sich eine Grundtendenz erkennen, bei einer umfassenden Reform des Nizza-Vertrages auch (oder vor allem) die institutionellen Fragen klären zu wollen. Allerdings wird etwa von polnischer Seite gerade diese Notwendigkeit bestritten und eine Fokussierung auf die inhaltlichen Fragen angemahnt. Auch der tschechische Präsident Vaclav Klaus sieht keine Notwendigkeit einer grundsätzlichen Änderung der institutionellen Regelungen von Nizza. ${ }^{42}$ Der Vorsitzende der französischen UMP Nicolas Sarkozy wiederum schlug jüngst vor, die Reform nicht in einem Schritt, sondern in drei Phasen zu vollziehen. Nach einer ersten möglichst zeitnah anzusetzenden institutionellen Reform sollen ebenfalls zeitnah bestimmte inhaltliche Bereiche über das Modell der verstärkten Zusammenarbeit angegangen werden. Eine grundlegende Überarbeitung des gesamten Vertragswerkes soll aber erst in einem später erfolgenden dritten Schritt angestrebt werden, wobei er hierzu die Einberufung eines neuen konventsähnlichen Gremiums vorsieht. ${ }^{43}$ Allerdings würde auch diese Phaseneinteilung die verhandlungstechnische Trennung von Sach- und Institutionenfragen und damit die zuvor beschriebenen Schwierigkeiten einer reinen Institutionenreform mit sich bringen.

Das Grundproblem aller Vorschläge zu einer umfassenden Reform des Vertrages von Nizza besteht darin, dass nicht ersichtlich ist, welches Ergebnis die hierzu notwendigen Neuverhandlungen haben werden und ob es leichter von allen 25 Mitgliedstaaten ratifiziert würde als der Verfassungsvertrag. Angesichts der Fülle der verschiedenen inhaltlichen Vorstellungen über die erforderlichen Reformen (Institutionen, auswärtiges Handeln, Innenund Justizpolitik, Energiepolitik, soziale Dimension) wäre man bei den Verhandlungen über eine solche Nizza-Reform zudem mit ähnlichen Problemen konfrontiert wie bei der oben beschriebenen Option einer umfassenden Reform des Verfassungsvertrages.

\section{Nizza plus ,Context Change" als kurzfristige Strategie}

Die letzte Variante, die auf dem Vertrag von Nizza basiert, läuft auf eine Beibehaltung der dortigen Vertragsgrundlage hinaus. Zwar sind die Experten sich einig, dass eine erweiterte Union mit den Verfahrensvorschriften von Nizza nur sehr eingeschränkt handlungsfähig sein wird. ${ }^{44}$ Allerdings bilden die bisherigen Erfahrungen mit dem Nizza-Regelwerk noch keine ausreichende empirische Grundlage, um diese These hinreichend verlässlich bestätigen zu können.

38 Vgl. ebenda; Chirac, Allocution lors de la présentation des vœux du Corps diplomatique.

39 Vgl. Polen will mit der Reform der EU ganz vorne beginnen, in: Frankfurter Allgemeine Zeitung vom 8. März 2006.

40 Vgl. Deutschland und Frankreich für Energie-Initiative, in: Frankfurter Allgemeine Zeitung vom 15. März 2006.

41 Vgl. Kapitel III, Abschnitt 10 des Vertrags über eine Verfassung für Europa.

42 Vgl. Andrew Rettman: Polish Ruling Party Says EU Consitution Is "Dead", in: Euobserver.com vom 16. Januar 2006; Czech, Polish Presidents Aren't Fan of Old EU Consititution, in: Pravda (englische Version) vom 17. Februar 2006. Vgl. Präsidenten Tschechiens und Polens gegen ein vereintes Europa, in: Euractiv vom 21. Februar 2006.

43 Vgl. Sarkozy, Vortrag vom 16. Februar 2006 in Berlin, S. 6.

44 Zur Bewertung des Vertrages von Nizza vgl. Mathias Jopp/Barbara Lippert/Heinrich Schneider (Hrsg.): Das Vertragswerk von Nizza und die Zukunft der Europäischen Union, Bonn 2001. 
In der aktuellen Diskussion können am ehesten die Äußerungen von Premierminister Blair als Plädoyer für ein - zumindest vorübergehendes - Festhalten am Vertrag von Nizza gesehen werden. Dies spricht er zwar nicht offen aus, prinzipiell läuft seine Äußerung, dass ,the issue at present is not the long term vision, but the short term strategy ${ }^{\text {“45 }}$ aber darauf hinaus. Denn unter einer solchen ,,short term strategy“ versteht er die Rückgewinnung der Akzeptanz bei den Bürgern durch eine effektive Politik, was er innerhalb des bestehenden Vertragswerkes erreichen möchte. Zwar erkennt er die Notwendigkeit tiefer gehender Reformen an, diese sollten jedoch eher in langfristiger Perspektive angegangen werden. ${ }^{46}$ Kurzfristig so kann man seine These zusammenfassen - solle man den Vertrag von Nizza mit Leben erfüllen.

Auch wenn Tony Blair auf der Spitzenebene der politischen Diskussion mit diesem Ansatz singulär erscheint, so steht er doch für ein Denken, das zumindest hinter vorgehaltener Hand in verschiedenen nationalen Ministerien immer wieder zu hören ist: nämlich die Diskussion über die Reform des Primärrechts zu vertagen und statt dessen nach Reformen und , better policies' innerhalb des bestehenden Vertragsrahmens zu streben. Der Vorteil dieses Ansatzes, so die Annahme, läge darin, dass sich auf diesem Wege praktische Fortschritte erzielen ließen. Wenn eine erfolgreiche Ausschöpfung des Nizza-Vertrages gelänge - so die Erwartung - ließe sich ein günstigeres Umfeld für ambitioniertere Projekte schaffen. In dieser Perspektive wäre der Ansatz, vor allem politikrelevante und kleinere institutionelle Verbesserungen unterhalb der Schwelle von Primärrechtsveränderungen durchzuführen, mittelfristig sowohl mit einer umfassenden Reform des Vertrages von Nizza als auch einer Wiederbelebung des Verfassungsprozesses vereinbar. ${ }^{47}$ Der Nachteil dieser Variante besteht allerdings darin, dass die Umsetzung bestimmter inhaltlicher Aspekte (zum Beispiel der Energiepolitik) für Staaten mit diesbezüglichen besonderen Interessen den Anreiz zu einer umfassenden Reform verringern könnte. Auch würde die Gefahr bestehen, dass auf den vielzitierten ,context change' der Debatte über die Zukunft der Union (mit höherer Akzeptanz bei den Bürgern durch erfolgreiche Politik auf der Basis eines unzureichenden Vertrags) Jahre, wenn nicht sogar weit mehr als ein Jahrzehnt gewartet werden müsste. Bei Blair ist zudem das Problem, dass er die Verfassung als abgehobene institutionelle Debatte ,in the room at the top of the tower" abqualifiziert und seine Vision ein ziemlich altbackenes britisches Verständnis einer ,ever closer union of nation states, cooperating, as of sovereign right, where it is in their interest to do so" beinhaltet. Außerdem will er erst nach dem Erfolg der „short term strategy“ nicht zum Verfassungsvertrag selbst, sondern zu den ,issues around the European Constitution“ zurückkehren, was immer dies beinhalten und verfahrensmäßig heißen mag.

45 Vgl. Blair, Speech on future of Europe.

46 Vgl. Die Verfassung ist nicht tot. Interview mit Tony Blair, in: Frankfurter Allgemeine Zeitung vom 17. Februar 2006.

47 Vgl. Wolfgang Wessels: Deutsche Europapolitik - Strategien für einen Wegweiser: Verstärkter Nutzen durch verbesserte Integration?, in: ders. und Udo Diedrichs (Hrsg.): Die neue Europäische Union: im vitalen Interesse Deutschlands? Studie zu Kosten und Nutzen der Europäischen Union für die Bundesrepublik Deutschland, Netzwerk Europäische Bewegung Deutschland und Europa Union Deutschland, Berlin 2006, S. 135-159; Mathias Jopp/Gesa-S. Kuhle: Wege aus der Verfassungskrise - die EU nach den gescheiterten Referenden in Frankreich und den Niederlanden; in: integration 3/2005, S. 261 und Mathias Jopp/Saskia Matl: Der Europäische Verfassungsvertrag als Höhepunkt im Prozess der Konstitutionalisierung der EU - eine Einführung, in: Jopp/Matl, Der Vertrag über eine Verfassung für Europa, S. 15-41. 


\section{Kurze Verfassung als Kopfvertrag zu Nizza - die kombinierte Strategie}

Eine mittlere Position zwischen dem Festhalten am Verfassungsvertrag und der Rückbesinnung auf den Vertrag von Nizza stellt die dritte Variante dar, die beide Vertragswerke miteinander kombiniert. Ausgangspunkt dieses Denkansatzes ist es, dass sich in der öffentlichen Diskussion der schiere Umfang des Verfassungsvertrages gemessen an dem ursprünglichen Anspruch des Verfassungsprojekts, das Primärrecht für die Bürger transparenter zu gestalten, immer wieder als Problem darstellt. Bereits vor Beginn der Konventsberatungen wurde deshalb diskutiert, ob es nicht sinnvoller wäre, die Verfassung als „Kopfvertrag“ über die bestehenden Verträge zu stellen. ${ }^{48}$ Dies hätte den Vorteil, dass sie ein überschaubares Dokument wäre, das die Grundsätze und Ziele der Union, die Inkorporation der Grundrechtecharta und den institutionellen Aufbau regelt. Hierdurch wäre auch vermieden worden, dass in den Referenden der gesamte Besitzstand der EU zur Abstimmung gestellt wurde. Nachteil eines solchen Kopfvertrages wäre allerdings, dass dieser ohne eine grundlegende Überarbeitung des bestehenden Primärrechts zahlreiche Inkonsistenzen mit sich bringen würde, die durch entsprechende Generalklauseln (,Alle dem Kopfvertrag widersprechenden Regelungen der bestehenden Verträge werden aufgehoben") allenfalls abgemildert werden könnten. Auch würde die Gesamtstruktur des europäischen Integrationssystems durch einen solchen zusätzlichen Vertrag weiter verkompliziert. Deshalb hat der Konvent sich schließlich gegen diese Variante entschieden, wobei auch die mit der Humboldt-Rede Joschka Fischers eingeführte Formel der ,konstitutionellen Neugründung “49 zur Favorisierung einer Neuordnung des gesamten Primärrechts beigetragen haben dürfte.

Nach dem Scheitern der Referenden in Frankreich und den Niederlanden hat das Konzept eines Kopfvertrages wieder an Attraktivität gewonnen. ${ }^{50}$ Denn, so die Idee, wenn man den dritten Teil aus der Verfassung ausklammern, sprich hier die bisherigen Verträge fortbestehen lassen und statt dessen die Verfassung nur auf die , wirklichen Verfassungsfragen' beschränken könnte, hätte man ein übersichtliches Dokument, das bei der Bevölkerung leichter auf Akzeptanz stoßen würde. Auch könnte hierdurch deutlicher gemacht werden, dass die Verfassung kein so fundamentaler Einschnitt ist wie dies in der öffentlichen Meinung wahrgenommen wird. Mit anderen Worten besteht dieses Konzept also daraus, die Verfassung auf ihren ersten und zweiten Teil mit den Grundrechten zu beschränken und als Kopfvertrag über die bisherigen Verträge zu stellen. Problematisch ist allerdings, dass der erste Teil inhaltlich zwar weitgehend dem Konzept eines Kopfvertrages entspricht, der Form nach aber nicht auf die bestehenden Verträge, sondern den dritten Teil der Verfassung abgestimmt ist, der zwar in der Substanz auf den bisherigen Verträgen beruht, aber doch einige wesentliche Änderungen in den Politikbereichen beinhaltet. Auch haben sich einige Begrifflichkeiten geändert: So spricht der erste Teil beispielsweise von Rahmengesetzen und Gesetzen, während die bestehenden Verträge die Termini Richtlinie und Verordnung verwenden. Dies würde zu zahlreichen Inkonsistenzen führen. Deshalb bedürfte es entweder einer grundlegenden Überarbeitung des ersten Teils oder aber einer umfassenden Anpassung der bestehenden Verträge. Beides würde allerdings die Einberufung einer neuen Regierungskonferenz und neue Ratifizierungsverfahren erforderlich machen, wobei völlig offen bliebe, ob es zu tragfähigen

48 Vgl. Daniel Göler: Die Europäische Union vor ihrer Konstitutionalisierung. Eine Bilanz der ersten Verfassungsentwürfe, in: integration 1/2003, S. 18.

49 Vgl. Joschka Fischer: Vom Staatenverbund zur Föderation, abgedruckt in: Wilfried Loth: Entwürfe einer europäischen Verfassung. Eine historische Bilanz, Bonn 2002, S. 249.

50 So hat das ehemalige Konventsmitglied Jürgen Meyer bei verschiedenen Gelegenheiten entsprechende Vorschläge unterbreitet. 
Ergebnissen käme. Zudem ist zu bezweifeln, ob der gewünschte Effekt des KopfvertragKonzepts, die Akzeptanz bei der Bevölkerung durch eine ,schlankere Verfassung' zu erhöhen, im Nachgang noch erreichbar ist.

\section{Flexible Integration}

Spätestens seit der Veröffentlichung des so genannten Schäuble-Lamers-Papiers $1994^{51}$ ist es fast eine Grundfeste der Europäischen Integration, dass in Krisenzeiten verschiedene Modelle der flexiblen Integration als Lösungsvorschläge unterbreitet werden. In der Verfassungsdebatte wurde bereits im Vorfeld der gescheiterten Referenden erörtert, im Falle einer fehlgeschlagenen Ratifizierung auf solche Modelle zurückzugreifen. Hintergrund war die Überlegung, dass in der erweiterten Union einheitliche Lösungen nur noch schwer erreichbar sein würden. Gerade im wissenschaftlichen Bereich wurde die Option diskutiert, dass die Europäische Union in Zukunft aus einem „Kern“, das heißt den Ländern, die den Verfassungsvertrag ratifiziert haben, und den übrigen Staaten besteht, für die der Vertrag von Nizza oder aber eine andere Vertragsgrundlage gelten könnte. ${ }^{52}$ Allerdings war unausgesprochene Voraussetzung dieser Überlegungen, dass die Verfassung in den , wichtigsten Staaten' der EU angenommen würde.

Mit dem gescheiterten Referendum in Frankreich hat diese Diskussion sich insoweit verschoben, als Modelle der flexiblen Integration nun nicht mehr als Option zur Umsetzung des Verfassungsvertrages gesehen werden, sondern als Möglichkeit, alternative Reformoptionen zu entwickeln. So hat der französische Präsident Chirac sich für die Bildung einer Pioniergruppe ausgesprochen, ${ }^{53}$ ohne allerdings auszuführen, was genau die inhaltlichen Zielsetzungen dieser Gruppe sein sollen. Dass Chirac hierbei Frankreich wie selbstverständlich als Mitglied einer solchen Pioniergruppe sieht, mutet angesichts der aktuellen Situation, in der vor allem Frankreich den gesamten Verfassungsprozess blockiert, bestenfalls als eigenwillig an. Deutlich konkreter sind die Vorstellungen des belgischen Premierministers Guy Verhofstadt, der die Bildung einer föderalen Europäischen Union fordert. ${ }^{54}$ Da ein solcher Vertiefungsschritt nicht von allen Mitgliedstaaten getragen werden könne, sollte dieses Europa nur von einem Teil der Staaten verwirklicht werden, wobei er hier die Euro-Gruppe als Ausgangspunkt nimmt. Die übrigen dreizehn Staaten sollten sich in einem weiteren Verbund an diese Föderation anschließen und jederzeit das Recht haben, dem „Integrationskern“ beizutreten.

Weg von dem Modell des Kerneuropa beziehungsweise der Pioniergruppe gehen die Überlegungen von Nicolas Sarkozy, der eher eine an dem Modell der variablen Geometrie orientierte sachbezogene Zusammenarbeit der dazu fähigen und willigen Staaten andenkt, ${ }^{55}$ wobei er als Beispiele hierfür den Bereich der Innen- sowie der Sicherheits- und Verteidigungspolitik nennt. ${ }^{56}$ Sarkozys Verweis etwa auf die Sicherheits- und Verteidigungspolitik hat dabei einen reellen Hintergrund, da sich hier in den letzten Jahren eine ganze Reihe von Formen flexibler Zusammenarbeit im Rüstungsbereich (z.B. OCCAR, Verteidigungsagentur), beim Konzept der Gefechtsverbände (Battle Groups) oder bei militärischen Missionen

51 Zur inhaltlichen Bewertung vgl. Daniel Göler: Europapolitik im Wandel. Deutsche Integrationsmotive und Integrationsziele nach der Wiedervereinigung, Münster 2004, S. 65-71.

52 Vgl. Heinrich Schneider: „Kerneuropa“. Ein aktuelles Schlagwort und seine Bedeutung, EI Working Papier Nr. 54, Wien 2004, S. 28.

53 Vgl. Chirac, Allocution lors de la présentation des vœux du Corps diplomatique.

54 Vgl. Verhofstadt, Die Vereinigten Staaten von Europa.

55 Vgl. Sarkozy, Vortrag vom 16. Februar 2006 in Berlin, S. 4.

56 Vgl. ebenda. 
herausgebildet haben. Hierbei handelt es sich jedoch nicht um die Nutzung des primärrechtlichen Instruments der verstärkten Zusammenarbeit, von dem die Sicherheits- und Verteidigungspolitik im Vertrag von Nizza ausgenommen wurde. Vielmehr handelt es sich um verschiedene Formen intergouvernementaler Zusammenarbeit zwischen Gruppen von Mitgliedstaaten oder auch allen Mitgliedstaaten im Rahmen der Union oder außerhalb von ihr. Mit dieser Art Zusammenarbeit könnte es zwar mehr oder weniger erfolgreich weiter gehen, Fragen des Primärrechts sind damit allerdings nicht gelöst.

Primärrechtlich gesehen bestand das Grundproblem der Diskussionen über Modelle flexibler Integration in der Vergangenheit darin, dass es an der konkreten Umsetzung mangelte. Dies zeigt sich nicht zuletzt auch daran, dass die nach den bestehenden Verträgen gegebenen Möglichkeiten der verstärkten Zusammenarbeit bisher nicht ausgeschöpft wurden. Außerdem sollte nicht außer Acht gelassen werden, dass die Modelle der verstärkten $\mathrm{Zu}-$ sammenarbeit von ihrem ursprünglichen Ansatz her hauptsächlich die Zielsetzung hatten, konkrete Probleme innerhalb bestimmter Politikbereiche zu überwinden. Ersatz für eine umfassende Reform des Primärrechts können sie kaum sein. Lediglich das Modell eines Kerneuropa beziehungsweise der Pioniergruppe würde sich hierfür anbieten. Angesichts der Widerstände, die solchen Überlegungen entgegengebracht wurden und werden, darf jedoch bezweifelt werden, dass dies ein Weg aus der aktuellen Krise sein könnte.

\section{Die weiteren Schritte}

Ungeachtet der konkreten inhaltlichen Bewertung des Verfassungsvertrages liegt ein Großteil der zur Zeit geäußerten Kritik in der ungünstigen innen- und europapolitischen Gesamtsituation in etlichen Mitgliedstaaten begründet. Viel spricht deshalb zunächst durchaus dafür, durch eine bessere Politik auf EU-Ebene und eine bessere öffentliche Vermittlung dieser Politik in die Richtung einer Stimmungsänderung zu arbeiten, ohne freilich parallel dazu die Verfolgung der Verfassungsfrage aus den Augen zu verlieren. Vor einer endgültigen Entscheidung über das weitere Vorgehen bleibt abzuwarten, wie und in welcher Form insbesondere Frankreich und die Niederlande den Verfassungsprozess wieder aufnehmen wollen. Da in beiden Ländern im nächsten Jahr Parlamentswahlen (und in Frankreich zudem Präsidentschaftswahlen) anstehen, ist eine Klärung dieser Situation vor Mitte 2007 nicht zu erwarten.

Zum Ablauf der gegenwärtigen Reflexionsphase am Ende der österreichischen Präsidentschaft wäre zunächst einmal eine Road Map zu verabschieden, die unter anderem die Verlängerung der Reflexionsphase, eine Zwischenbilanz zum weiteren Ratifizierungsprozess am Ende der finnischen Ratspräsidentschaft und konkrete Vorschläge zum weiteren Verfahren am Ende der deutschen Vorsitzperiode vorsieht. Um bis dahin eine gewisse Reformdynamik zu erhalten, wäre es förderlich, den Kreis der Ratifizierungsstaaten zu vergrößern, um für den Fall nicht auszuschließender späterer Einzelverhandlungen die Grundlagen, von denen auszugehen ist, durch eine große Mehrheit von Mitgliedstaaten von vornherein zu definieren und abzusichern. Darüber hinaus sollten nach den Wahlen in Frankreich und den Niederlanden zunächst einmal Strategien und Optionen ausgelotet werden, wie den Bedürfnissen derjenigen Mitgliedstaaten und ihrer Bevölkerungen entgegenzukommen ist, die den Verfassungsvertrag abgelehnt haben oder bei denen eine negative Entscheidung droht (zum Beispiel durch Protokolle oder ergänzende Erklärungen). Und schließlich wäre zu prüfen, inwieweit statt des sich als schwierig erwiesenen Wegs einzelstaatlicher Referenden die jeweilige parlamentarische Ratifizierung des Verfassungsvertrages angestrebt werden kann. Ausgenommen blieben hiervon jene Länder, bei denen ein Referendum verfassungsmäßig 
vorgeschrieben ist oder einen Ausweg aus einer voraussehbaren parlamentarischen Blockade oder Ablehnung bieten könnte.

Welche darüber hinausgehende Strategie zur Lösung der gegenwärtigen Krise letztlich gewählt wird, ist zum jetzigen Zeitpunkt noch nicht abzuschätzen. Diejenigen, die für ein „Über-Bord-Werfen“ der Verfassung plädieren, verweisen darauf, dass es unmöglich wäre, diese in allen Mitgliedstaaten zu ratifizieren. Diejenigen, die am Verfassungsvertrag festhalten wollen, führen zu Recht an, dass dieser ein Kompromiss ist, der durch den Konvent und die Regierungskonferenz ausgehandelt, von allen 25 Staats- und Regierungschefs feierlich paraphiert und bis jetzt von 14 Mitgliedstaaten ratifiziert wurde; aller Voraussicht nach werden weitere Staaten folgen. Zudem bergen in ihren Augen alle zur Zeit diskutierten Alternativmodelle zum Verfassungsvertrag - wie im Vorangehenden dargelegt - nicht unerhebliche Risiken in sich. Nicht zuletzt deshalb erscheint es durchaus sinnvoll, einerseits die Ratifizierung des Verfassungsvertrags offen zu halten und andererseits an der Verbesserung der europapolitischen Rahmenbedingungen zu arbeiten. Selbst wenn eine hierdurch bedingte längere Zeit des Wartens auch über 2007 hinaus (möglicherweise bis zur Neuwahl des EP im Jahre 2009) unbefriedigend sein mag, gilt jedoch gerade in der gegenwärtigen Lage die kürzlich von Angela Merkel in anderem Zusammenhang gemachte Äußerung, dass man in der Politik nicht immer gleich alle Varianten ausdiskutieren und sofort Lösungen finden, sondern ,auch mal die Nerven behalten ${ }^{“ 57}$ muss.

Dieser Beitrag entstand im Rahmen des Forschungsprojekts „Ein Europa der Bürger Verfassung und effiziente Politik“ der ASKO EUROPA-STIFTUNG und des Instituts für Europäische Politik (IEP).

57 Klaus-Dieter Frankenberger/Stephan Löwenstein: „Auch mal die Nerven behalten“. Lob für Merkel in München, in: Frankfurter Allgemeine Zeitung vom 6. Februar 2006. 